\title{
The elevated serum parathyroid hormone level is associated with the occurrence of atrial fibrillation in patients with advanced heart failure
}

Joanna Wiśniewska, Anna Drohomirecka, Natalia Wiligórska, Małgorzata Sobieszczańska-Małek, Tomasz Zieliński

Department of Heart Failure and Transplantology, The Cardinal Stefan Wyszyński National Institute of Cardiology, Warsaw, Poland

Correspondence to: Natalia Wiligórska, MD Department of Heart Failure and Transplantology, The Cardinal Stefan Wyszyński National Institute of Cardiology, ul. Alpejska 42, 04-628 Warszawa, Poland, phone: +48223434483 email: nwiligorska@ikard.pl Received: September 9, 2020. Revision accepted: October 19, 2020. Published online: October 27, 2020. Kardiol Pol. 2020; 78 (12): 1274-1277 doi:10.33963/KP.15667 Copyright by the Author(s), 2020
Introduction In the past decade, the role of calcium and phosphate metabolism in the pathogenesis of cardiovascular disease attracted growing scientific interest, which has resulted in an increasing number of studies on this issue. Numerous authors have investigated the link between vitamin $\mathrm{D}$ and parathormone (PTH) status and the risk of atrial fibrillation (AF) in the general population. Although a meta-analysis by Zhang et $\mathrm{al}^{1}$ has shown that vitamin $\mathrm{D}$ deficiency can be associated with the incidence of $\mathrm{AF}$, the results of a recent large observational study ${ }^{2}$ have implied that PTH is a better predictor of a new AF onset. Moreover, both vitamin D deficiency and increased parathormone levels are thought to be related to disease severity and to predict worse prognosis in patients with heart failure (HF). ${ }^{3}$

Considering that $\mathrm{AF}$ is the most common arrhythmia in patients with HF and the fact that there is no sufficient data on the relationship between PTH/vitamin D imbalance and AF in this group of patients, we decided to analyze the parameters of calcium-phosphate metabolism in relation to cardiac rhythm in patients with advanced HF.

Methods The study group consisted of 138 clinically stable patients ( $83 \%$ men, $17 \%$ women) admitted to the Department of Heart Failure and Transplantology due to chronic HF with reduced ejection fraction. The study patients were divided into 2 groups according to the type of cardiac rhythm, as confirmed by an electrocardiogram on admission: sinus rhythm or AF. All patients in the AF group presented with permanent arrhythmia. Five patients had undergone ablation for $\mathrm{AF}$, a single patient for atrial flutter, and 4 of those patients maintained sinus rhythm during the study. Exclusion criteria were as follows: a creatinine level above $2.5 \mathrm{mg} / \mathrm{dl}$, primary hyperparathyroidism, vitamin D and calcium supplementation, cancer (active, present in a medical history, or suspected), and treatment for osteoporosis. Serum concentrations of 25-hydroxyvitamin D, PTH, calcium, and phosphorus were measured during the first days of hospitalization. The study was approved by the local ethics committee and all patients provided informed consent prior to enrollment.

Statistical analysis The Shapiro-Wilk test was used to verify the null hypothesis of normal data distribution. Continuous variables were expressed as mean (SD) in the case of values representing normal distribution or median (interquartile range) in the case of values with non-normal distribution. Differences between the study groups were tested using the $t$, Mann-Whitney, or $\mathrm{X}^{2}$ tests (with the Yates correction for continuity where needed), as appropriate. The relationship between continuous variables was evaluated by the Spearman correlation test. Logistic regression analysis was performed in order to identify the independent predictors of AF incidence. The multivariable model included variables identified as significant in the univariate model. A $P$ value less than 0.05 was considered significant. Statistical analysis was conducted using the Statistica PL 12.0 software (StatSoft Inc., Palo Alto, California, United States).

Results and discussion Atrial fibrillation was diagnosed in 26 patients (19\%). The baseline characteristics of the study patients and 
TABLE 1 Baseline characteristics and test results of the study population

\begin{tabular}{|c|c|c|c|c|}
\hline Characteristics & All patients $(n=138)$ & $\operatorname{AF}(+)(n=26)$ & $\operatorname{AF}(-)(n=112)$ & $P$ value \\
\hline \multicolumn{5}{|l|}{ Clinical characteristics } \\
\hline Age, y, median (IQR) & $52.3(42.7-58.5)$ & $58.6(43.7-64.4)$ & $50(42-57.4)$ & 0.002 \\
\hline Male sex & $114(83)$ & $21(81)$ & $119(83)$ & 0.99 \\
\hline BMI, kg/m², mean (SD) & $27.7(3.9)$ & $28.7(3.3)$ & $27.5(4.1)$ & 0.13 \\
\hline Coronary artery disease & $45(33)$ & $5(19)$ & $40(36)$ & 0.11 \\
\hline DCM & $93(67)$ & $21(81)$ & $72(64)$ & 0.11 \\
\hline Hypertension & $39(28)$ & $9(35)$ & $30(27)$ & 0.43 \\
\hline Diabetes & $29(21)$ & $3(12)$ & $26(23)$ & 0.19 \\
\hline \multirow[t]{2}{*}{ NYHA class } & $97(70)$ & $18(69)$ & $79(70.5)$ & \multirow[t]{2}{*}{0.9} \\
\hline & $41(30)$ & $8(31)$ & $33(29)$ & \\
\hline \multicolumn{5}{|l|}{ Echocardiographic parameters } \\
\hline LVEF, \%, median (IQR) & $28(21-35)$ & $25(20-35)$ & $28.5(22-35)$ & 0.94 \\
\hline $\mathrm{LAA}, \mathrm{cm}^{2}$, median (IQR) & $28(22.5-36)$ & $36.3(28-34)$ & $26(22-32.9)$ & $<0.001$ \\
\hline Mitral regurgitation ${ }^{\mathrm{a}}$ & $35(25)$ & $9(35)$ & $26(23)$ & 0.23 \\
\hline Tricuspid regurgitationa & $11(80)$ & $4(15)$ & $7(6)$ & 0.25 \\
\hline \multicolumn{5}{|l|}{ Laboratory test results } \\
\hline NT-proBNP, pg/ml, median (IQR) & $915(410-1860)$ & $1273(911-2290)$ & $789(353-1650)$ & 0.008 \\
\hline eGFR, $\mathrm{ml} / \mathrm{min} / 1.73 \mathrm{~m}^{2}$, mean (SD) & $104(30)$ & $94(25)$ & $106(31)$ & 0.05 \\
\hline $\mathrm{Ca}, \mathrm{mmol} / \mathrm{l}$, mean (SD) & $2.4(0.1)$ & $2.4(0.1)$ & $2.4(0.1)$ & 0.83 \\
\hline $\mathrm{P}, \mathrm{mmol} / \mathrm{l}$, mean $(\mathrm{SD})$ & $1.1(0.2)$ & $1.1(0.1)$ & $1.2(0.2)$ & 0.31 \\
\hline Vitamin D, ng/ml, median (IQR) & $14.8(10.1-21.9)$ & $18.6(13.4-23.5)$ & $14(9.7-20.6)$ & 0.04 \\
\hline PTH, pg/ml, median (IQR) & $46.9(36.9-66.6)$ & $69.5(56-93)$ & $43.8(35.1-58.5)$ & $<0.001$ \\
\hline \multicolumn{5}{|l|}{ Medication } \\
\hline$\beta$-Blockers & $137(99)$ & $26(100)$ & $111(99)$ & 0.42 \\
\hline ACEI/ARB & $136(99)$ & $26(100)$ & $110(98)$ & 0.82 \\
\hline Spironolactone/ eplerenone & $125(91)$ & $23(88)$ & $102(91)$ & 0.97 \\
\hline Loop diuretics & $114(83)$ & $22(85)$ & $92(82)$ & 0.99 \\
\hline Amiodarone & $16(12)$ & $3(12)$ & $13(12)$ & 0.74 \\
\hline Digoxin & $24(17)$ & $7(27)$ & $17(15)$ & 0.26 \\
\hline \multicolumn{5}{|l|}{ Exercise capacity test results } \\
\hline 6-minute walk test, m, median (IQR) & $470(400-520)(n=132)$ & $420(340-480)(n=23)$ & $480(410-530)(n=109)$ & 0.01 \\
\hline Peak VO during CPX, $\mathrm{ml} / \mathrm{kg} / \mathrm{min}$, median (IQR) & $16(12.4-19)(n=114)$ & $12.1(10-16.5)(n=19)$ & $16(13-19.9)(n=95)$ & 0.008 \\
\hline
\end{tabular}

Data are presented as the number (percentage) of patients unless otherwise indicated.

a At least moderate regurgitation

SI conversion factors: to convert vitamin D to nmol/l, multiply by 2.5 , and parathormone to $\mathrm{pmol} / \mathrm{l}$, by 0.106 .

Abbreviations: -, absent; ACEI, angiotensin-converting enzyme inhibitor; AF, atrial fibrillation; ARB, angiotensin II receptor blocker; CPX, cardiopulmonary exercise testing; DCM, dilated cardiomyopathy; IQR, interquartile range; LAA, left atrial area; LVEF, left ventricular ejection fraction; NT-proBNP, N-terminal fragment of the prohormone brain natriuretic peptide; NYHA, New York Heart Association; $\mathrm{VO}_{2}$, oxygen uptake; +, present

differences in relation to this arrhythmia are presented in TABLE 1 .

The parameters listed in TABLE1 were analyzed by means of univariate regression analyses, except the results of exercise capacity tests. Advanced age, an enlarged left atrial area, and elevated PTH levels were identified as the predictors of AF. All of the following factors remained significant when included in the multivariable regression analysis model: age (odds ratio [OR], 
1.06 [per 1 year]; 95\% CI, 1-1.11; $P=0.04$ ), left atrial area $\left(\mathrm{OR}, 1.09\right.$ [per $1 \mathrm{~cm}^{2}$ ]; $95 \% \mathrm{CI}$, 1.03-1.15; $P=0.001)$, and the PTH level (OR, 1.02 [per $1 \mathrm{pg} / \mathrm{ml}$ ]; 95\% CI, 1.01-1.04; $P=0.002$ ).

Certain processes occurring in the course of $\mathrm{HF}$, such as increased aldosterone activity and diuretic-induced calcium loss, lead to the development of secondary hyperparathyroidism. Moreover, in the cases complicated by renal dysfunction, phosphorus retention and disturbances in 25-hydroxyvitamin D synthesis are observed. On the other hand, several mechanisms suggest that the imbalanced regulation of calcium-phosphate metabolism plays a role in the pathophysiology of HF progression. ${ }^{4}$ Receptors for both vitamin D and PTH are expressed in a wide range of tissues, including the myocardium and vessel walls. ${ }^{4,5}$ The reported effects of vitamin D deficiency and elevated PTH levels on the cardiovascular system include the stimulation of the reninangiotensin-aldosterone system, exacerbating tissue inflammation, and endothelial dysfunction. ${ }^{4}$ Additionally, PTH can induce myocardial hypertrophy and fibrosis as well as increase the heart rate. ${ }^{4}$ All those actions may also promote the electrical and structural remodeling of the atrium leading to $\mathrm{AF}$ development.

To date, the results of studies on the correlation among $\mathrm{AF}, \mathrm{HF}$, and calcium-phosphate metabolism have been equivocal, and there is insufficient evidence to draw firm conclusions. While Schierback et $\mathrm{al}^{3}$ did not find any differences with regard to $\mathrm{AF}$ incidence in terms of PTH levels, Belen et $\mathrm{al}^{6}$ reported that both vitamin D and PTH levels changed (decreased and elevated, respectively) in patients with $\mathrm{HF}$ and AF compared with those with sinus rhythm. Since vitamin D deficiency is the predominant cause of secondary hyperparathyroidism, it may be expected that low vitamin D and high PTH concentrations would be responsible for similar effects. Our study in patients with HF showed a relationship between $\mathrm{AF}$ and the elevated PTH level yet not with other parameters of calciumphosphate metabolism (vitamin D, calcium and phosphorus concentration). However, increased PTH levels correlated with the low levels of vita$\min \mathrm{D}(R=-0.3, P<0.001)$. It may suggest that the pleiotropic effects of PTH are seen before evident calcium and phosphate homeostasis disturbances become apparent and remain consistent with changes in vitamin D levels. Furthermore, similar to Altay et al, ${ }^{7}$ we observed that both AF (TABLE 1) and elevated PTH levels were associated with HF progression (PTH levels correlated with higher $\mathrm{N}$-terminal fragment of the prohormone brain natriuretic peptide levels $[R=0.3, P<0.001]$ and with poorer results of exercise tests [6-minute walk test: $R=-0.2$, $P=0.02$; cardiopulmonary exercise test: $R=$ $-0.4, P<0.001])$. In the light of the above observations, the relationship between PTH levels and $\mathrm{AF}$ seems to be more pronounced in patients with advanced HF.

The discussion of the abovementioned selected mechanisms is a contribution to the ongoing research, with numerous questions still to be explored. In addition to the fact that there is a link among AF, PTH levels, and HF, it should be emphasized that $\mathrm{PTH}$ is the main regulator of the serum calcium concentration. Therefore, it may be suspected that it also influences cardiac electrophysiology. Recently, a clinical study has demonstrated that adolescents with vitamin $\mathrm{D}$ deficiency were more prone to ventricular repolarization anomalies. ${ }^{8}$ Although it is tempting to apply the analogy to atrial arrhythmias, unfortunately, there have been no studies demonstrating the direct impact of PTH levels on the electrical activity of atrial myocytes.

Study limitations Patients included in our study were significantly younger and presented with more advanced HF (higher N-terminal fragment of the prohormone brain natriuretic peptide levels and lower left ventricular ejection fraction) than populations usually enrolled in such observational studies. Moreover, the measurements of vitamin D levels were not adjusted for the season.

Conclusions In our study, elevated PTH levels predicted the incidence of AF. Nevertheless, it remains controversial whether this metabolic disorder directly contributes to arrhythmia or there is just a coincidence of both pathologies in the clinical settings of advanced HF. However, if we consider the fact that the PTH level remained to be a significant predictor of $\mathrm{AF}$, in addition to well-supported clinical risk factors such as advanced age and an enlarged left atrium, its potential role in the pathophysiology of atrial arrhythmia cannot be neglected.

\section{ARTICLE INFORMATION}

ACKNOWLEDGMENTS The authors would like to thank Katarzyna Kodziszewska, $M D$, PhD, for medical proofreading.

\section{CONFLICT OF INTEREST None declared.}

OPEN ACCESS This is an Open Access article distributed under the terms of the Creative Commons Attribution-NonCommercial-NoDerivatives 4.0 International License (CC BY-NC-ND 4.0), allowing third parties to download articles and share them with others, provided the original work is properly cited, not changed in any way, distributed under the same license, and used for noncommercial purposes only. For commercial use, please contact the journal office at kardiologiapolska@ptkardio.pl.

HOW TO CITE Wiśniewska J, Drohomirecka A, Wiligórska N, et al. The elevated serum parathyroid hormone level is associated with the occurrence of atrial fibrillation in patients with advanced heart failure. Kardiol Pol. 2020; 78: 1274-1277. doi:10.33963/KP.15667

\section{REFERENCES}

1 Zhang Z, Yang Y, $\mathrm{Ng} \mathrm{CY}$, et al. Meta-analysis of vitamin D deficiency and risk of atrial fibrillation. Clin Cardiol. 2016; 39: 537-543.

2 Trevisan C, Piovesan F, Lucato $P$, et al. Parathormone, vitamin D and the risk of atrial fibrillation in older adults: a prospective study. Nutr Metab Cardiovasc Dis. 2019; 29: 939-945.

3 Schierbeck LL, Jensen TS, Bang U, et al. Parathyroid hormone and vitamin D markers for cardiovascular and all cause mortality heart failure. Eur J Heart Fail. 2011; 13: 626-632. 
4 Kolaszko A, Kubiak G, Nowalany-Kozielska E. Parathormone and vitamin d in heart failure - theoretical influence on disease development and potential therapy targets. Wiad Lek. 2017; 70: 804-811.

5 Ureña P, Kong XF, Abou-Samra AB, et al. Parathyroid hormone (PTH)/PTH-related peptide receptor messenger ribonucleic acids are widely distributed in rat tissues. Endocrinology. 1993; 133: 617-623.

6 Belen E, Aykan AC, Kalaycioglu E, et al. Low-level vitamin D is associated with atrial fibrillation in patients with chronic heart failure. Adv Clin Exp Med. 2016; 25: 51-57.

7 Altay $\mathrm{H}$, Zorlu A, Binici S, et al. Relation of serum parathyroid hormone level to severity of heart failure. Am J Cardiol. 2012; 109: 252-256.

8 Bagrul D, Atik F. Association of vitamin D deficiency with ventricular repolarization abnormalities. Kardiol Pol. 2019; 77: 853-858. 